\title{
Transcriptional regulation of Niemann-Pick C1-like 1 gene by liver receptor homolog-1
}

\author{
Eui Sup Lee, Hyun Jung Seo, Su Sun Back, Seung Ho Han, Yeon Ji Jeong, Jin Wook Lee, Soo Young Choi E Kyuhyung Han * \\ Department of Biomedical Science and Research Institute of Bioscience and Biotechnology, Hallym University, Chunchon 24252, Korea
}

\begin{abstract}
Factors that modulate cholesterol levels have major impacts on cardiovascular disease. Niemann-Pick C1-like 1 (NPC1L1) functions as a sterol transporter mediating intestinal cholesterol absorption and counter-balancing hepatobiliary cholesterol excretion. The liver receptor homolog 1 (LRH-1) had been shown to regulate genes involved in hepatic lipid metabolism and reverse cholesterol transport. To study whether human NPC1L1 gene is regulated transcriptionally by LRH-1, we have analyzed evolutionary conserved regions (ECRs) in HepG2 cells. One ECR was found to be responsive to the LRH-1. Through deletion studies, $\mathrm{LRH}-1$ response element was identified and the binding of LRH-1 was demonstrated by EMSA and ChIP assays. When SREBP2, one of several transcription factors which had been shown to regulate NPC1L1 gene, was co-expressed with LRH-1, synergistic transcriptional activation resulted. In conclusion, we have identified LRH-1 response elements in NPC1L1 gene and propose that LRH-1 and SREBP may play important roles in regulating NPC1L1 gene. [BMB Reports 2015; 48(9): 513-518]
\end{abstract}

\section{INTRODUCTION}

Factors that modulate circulating and tissue cholesterol levels have major impacts on cardiovascular disease. Cholesterol homeostasis in the body is tightly balanced by de novo biosynthesis, intestinal absorption, and biliary and fecal excretion. Niemann-Pick C1-Like 1 (NPC1L1) was found to play an important role in intestinal cholesterol absorption (1) and prevent extensive loss of cholesterol by reuptaking biliary cholesterol into hepatocytes (2). NPC1L1 is widely expressed in many human tissues, and it is highly expressed in the liver and small intestine (1). Naturally occurring mutations that disrupt NPC1L1 function were found to be associated with reduced plasma

${ }^{*}$ Corresponding author. Tel: +82-33-248-2115; Fax: +82-33-2563420; E-mail: khan@hallym.ac.kr

http://dx.doi.org/10.5483/BMBRep.2015.48.9.253

Received 26 November 2014, Revised 17 December 2014, Accepted 25 February 2015

Keywords: Cholesterol, LRH-1, NPC1L1, SERBP2, Transcription
LDL cholesterol levels and a reduced risk of coronary heart disease (3). NPC1L1 is a molecular target of ezetimibe, which is a pharmacological inhibitor of cholesterol absorption (4), and it has been used to treat hypercholesterolemia.

The nuclear receptor liver receptor homolog 1 ( $\mathrm{LRH}-1$ or NR5A2), a member of the NR5A superfamily of nuclear receptors, had been shown to be a determinant of reverse cholesterol transport and atherosclerosis susceptibility (5). The LRH-1 is expressed in endoderm-derived tissues such as the liver, pancreas, and intestine in adults and in the developing embryo (6). The hepatic LRH-1 had been shown to affect the expression of genes involved in hepatic reverse cholesterol transport, including scavenger receptor B type 1 (Scarb1) which is involved in HDL uptake into hepatocytes (7). The LRH-1 had also been shown to affect the expression of ABCG5 and ABCG8 genes which are important for cholesterol excretion into bile (8). Other established LRH-1 target genes in the liver are HDL formation, cholesterol exchange between lipoproteins, bile acid-synthesizing enzymes, bile salt export pump, and fatty acid synthesis (9-13). Since LRH-1 plays such a broad role in regulating genes involved in hepatic lipid metabolism and reverse cholesterol transport, it would be reasonable to raise a possibility that $\mathrm{LRH}-1$ affects hepatic expression of NPC1L1 gene. Several transcription factors involved in cholesterol metabolism take roles for the regulation of NPC1L1 gene. For example, hepatocyte nuclear factor $1 \alpha(\mathrm{HNF} 1 \alpha)$, hepatocyte nuclear factor $4 \alpha(\mathrm{HNF} 4 \alpha)$, peroxisome proliferatoractivated receptor $\alpha$ (PPAR $\alpha$ ), and SREBP2 have been shown to regulate NPC1L1 gene (14-17). However, there have been no reports on transcriptional regulation of NPC1L1 gene by LRH-1.

To identify the functional LRH-1 response element (LREs) of the NPC1L1 gene, we focused on the conserved regions determined by comparing the corresponding sequences of human and mouse genes using the evolutionary conserved region (ECR) browser (http://ecrbrowser.dcode.org). Six ECRs were selected and the corresponding DNA fragments have been cloned. The ECRs have been analyzed using the CMV-luciferase reporter system in HepG2 cells. As a result, we have identified LRH-1 response elements in NPC1L1 gene for the first time and propose that LRH-1, in combination with SREBP2, plays important roles in regulating NPC1L1 gene.

ISSN: 1976-670X (electronic edition)

Copyright (C) 2015 by the The Korean Society for Biochemistry and Molecular Biology

(c) This is an open-access article distributed under the terms of the Creative Commons Attribution Non-Commercial License (http://creativecommons.org/licenses/by-nc/4.0) which permits unrestricted non-commercial use, distribution, and reproduction in any medium, provided the original work is properly cited. 


\section{RESULT AND DISCUSSION}

Evolutionary Conserved Regions (ECRs) of NPC1L1 gene Human NPC1L1 gene maps to chromosome 7p13, spans 29 kb, encodes a $5 \mathrm{~kb} \mathrm{mRNA}$ and predominantly produces a protein of 1332 amino acids (18). In order to focus on the evolutionarily meaningful regions, genomic DNA sequence of the human NPC1L1 gene was compared with that of the corresponding mouse genes using the ECR browser (http://ecrbrowser.dcode. org). Evolutionary conserved regions (ECRs) with a minimum length of $200 \mathrm{bp}$ and a minimum identity of $58 \%$ were searched, which resulted in forty ECRs. We have selected six out of these forty by filtering out ECRs, where intron sequences take up less than $80 \%$ and where transcription start site, transposons, or simple repeats are found. Their positions in NPC1L1 gene are as follows: ECR1: $-4166 \sim-3800$, ECR2: $-2108 \sim$ -1729, ECR3: -924 -639, ECR4: +2580 + 2915, ECR5: +3322 + 3838, and ECR6: +29963 + 35425 .

LRH-1 nuclear receptor regulates NPC1L1 gene expression As a method to determine whether $\mathrm{LRH}-1$ induces endogenous NPC1L1 gene, HepG2 cells were transfected with LRH-1 expression plasmid. Overexpression of $\mathrm{LRH}-1$ induced the mRNA level of NPC1L1 gene (Fig. 1A, top panel). As expected, the overexpression of SREBP2, which had already been shown to regulate NPC1L1 gene (17), induced NPC1L1 gene as well. The overexpression of $\mathrm{LRH}-1$ and SREBP2 proteins were confirmed by immunoblotting (Fig. 1A, bottom panels).

\section{ECR3 responds to $\mathrm{LRH}-1$ nuclear receptor}

To determine which ECR contains LRH-1 response element, we first constructed six $\mathrm{pE}-\mathrm{CMV}$-LUC reporter plasmids by inserting each of the ECR fragments, amplified by the PCR reaction, into the pT-CMV-LUC plasmid which contains the cytomegalovirus promoter as a heterologous basal promoter (19). Then, we performed transient cotransfection assay by introducing each of the six pE-CMV-LUC reporter plasmids into HepG2 cells, along with the LRH-1 expression plasmid. There was LRH-1-dependent induction (by approximately 3-fold) of reporter activity when the ECR3 was tested (Fig. 1B).

Identification of a putative LRH-1 response element in ECR3 To map the $\mathrm{LRH}-1$ response element (LRE) in the ECR3, we made a series of truncations in the 286-bp ECR3 (-924 $-639)$. Truncation from the upstream end $(-924)$ down to -812 (pE3-d52-CMV-LUC; -812 -639) resulted in a small change in reporter activity, while further truncation down to -775 (pE3-d53-CMV-LUC; $-775 \sim-639$ ) resulted in a drastic decrease in LRH-1-dependent induction. This suggests that there is a LRE within the 37-bp region between -812 and -775 of the ECR3 (Fig. 1C). LRH-1 binds as a monomer to its response elements (6). By comparing with the consensus LRE, yCAAGgycr (20), we discovered two putative LREs (-807 CCAAGGTAG -799 and -800 AGGCCTTGA - 792) which
(A)

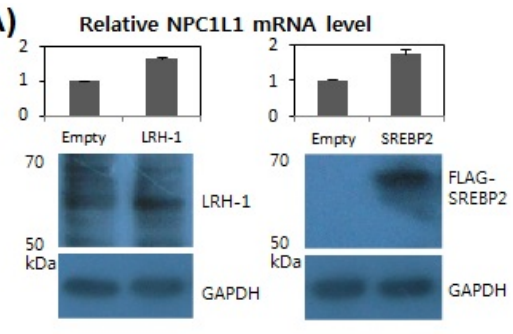

(B)
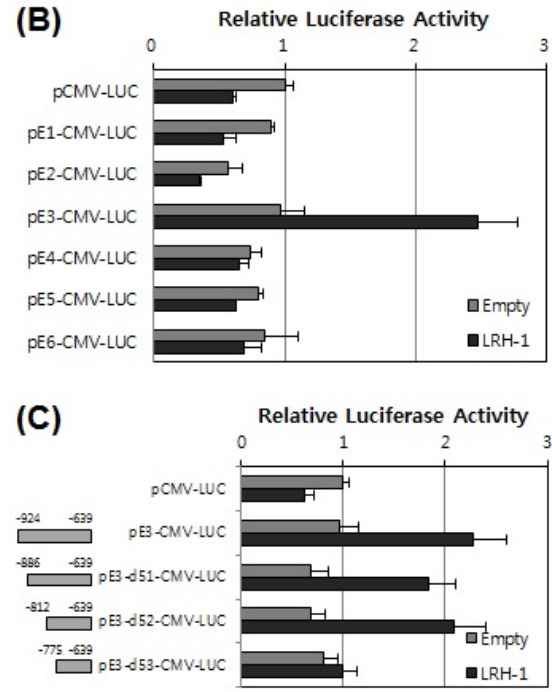

Fig. 1. LRH-1 nuclear receptor regulates NPC1L1 gene expression. (A) Transcriptional induction of NPC1L1 gene by LRH-1 and SREBP2 is shown. HepG2 cells in a 6-well plate were transfected with $0.2 \mu \mathrm{g}$ of LRH-1 or FLAG-tagged SREBP2 expression plasmid (total $3.3 \mu \mathrm{g}$ DNA) with CalFectin (SingaGen). Two days after transfection, the cells were harvested for isolation of total RNA and whole-cell extracts. Gene expression was analyzed by QRT-PCR, using primer sets specific for human NPC1L1 and GAPDH as an internal control (top). Aliquots $(40 \mu \mathrm{g})$ of protein were subjected to SDS-PAGE and immunoblot analysis using anti-LRH-1 (sc-25389X, Santa Cruz Biotech), anti-FLAG (200-301-383S, Rockland), and anti-GAPDH antibody (sc-32233) (bottom). (B) ECRs were tested for their responsiveness to the LRH-1 nuclear receptor using heterologous CMV promoter. Transfection with $10 \mathrm{ng}$ of LRH-1 expression plasmid was performed, as described in Materials and Methods. Relative luciferase activities were calculated by dividing the normalized luciferase activities by that of the control cells transfected with pCMV-LUC and an empty expression plasmid (pcDNA3). Values are expressed as the mean \pm S.E. $(2<n<8)$. (C) Deletion mapping of ECR3 for LRH-1 responsive elements is shown. On the left side is the schematic representation of the deletion series of the pE3-CMV-LUC reporter plasmid.

are in a reverse orientation to each other and overlapping by two base pairs (Fig. 2A). The two putative LREs have one nucleotide mismatch from the consensus sequence, at the eighth and ninth positions, respectively. The region $(-791 \sim-639)$ downstream of the putative LREs contains no other matching sequences to the consensus response element. 

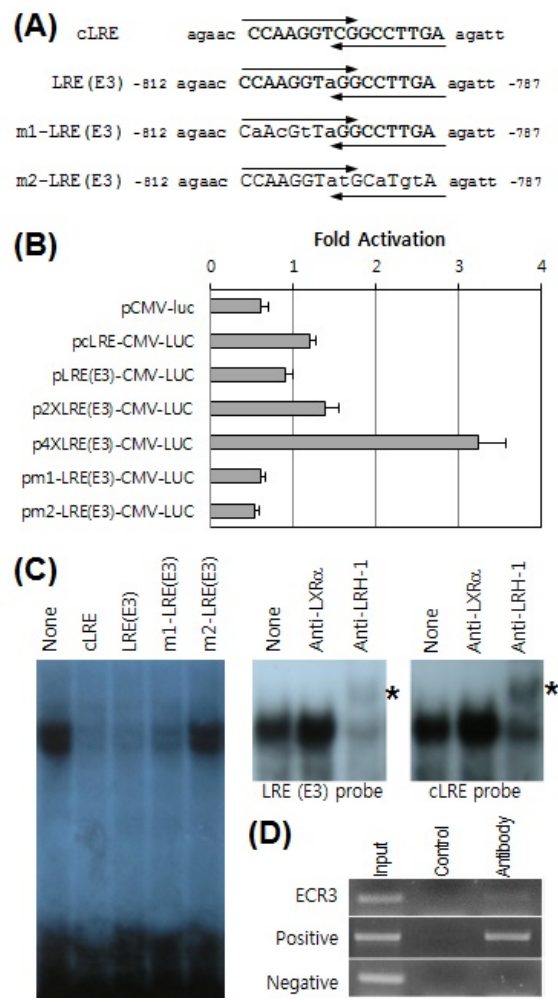

Fig. 2. Identification of $L R H-1$ response elements (LRE)s in ECR3 is shown. (A) Sequences of oligonucleotides containing putative and mutated LRH-1 response elements in ECR3 are shown. Nucleotide sequence of the $\operatorname{LRE}(\mathrm{E} 3)$ deviates from the consensus sequence by one nucleotide. (B) The putative $\mathrm{LRH}-1$ response element is required for transcriptional induction by $\mathrm{LRH}-1$. The fold of activation was calculated for each reporter construct by dividing normalized luciferase activity with LRH-1 expression by that with empty expression plasmid. Values are expressed as the mean \pm S.E. $(3<n<6)$. (C) EMSA demonstrates the binding of the putative $\mathrm{LRH}-1$ response element in the ECR3 region to $\mathrm{LRH}-1$ protein. DNA fragments containing the $\operatorname{LRE}(\mathrm{E} 3)$ and $\mathrm{CLRE}$ were labeled with ${ }^{32} \mathrm{P}$ and were used as a probe. The labeled probe was incubated with in vitro translated $\mathrm{LRH}-1$ protein with or without competitors. As competitors, 100-fold excess amounts of the unlabeled DNA fragments were used as marked above each lane. For the supershift assay, $2 \mu \mathrm{g}$ of each anti-LXR $\alpha$ (sc-1201X) or antiLRH-1 antibody (sc-25389X) was added in the binding reaction mixture thirty minutes prior to the addition of the probe. The supershifted complex is indicated with asterisk. (D) Chromatin immunoprecipitation assay is used to detect binding of LRH-1 to the LRE in ECR3. Immunoprecipitated chromatin was amplified by PCR using primers designed to amplify 161-bp region of ECR3 containing the LRE. For positive and negative controls, 288-bp and 177-bp regions of BSEP were amplified, respectively.

To prove that the putative LREs are required for transcriptional induction of NPC1L1 gene by LRH-1, we inserted a 26-bp DNA fragment $(-812 \sim-787)$ containing the putative elements into the pT-CMV-LUC. For an unknown reason, the expression of $\mathrm{LRH}-1$ resulted in the reduction of reporter activity (down to about $60 \%$ compared to that with empty expression plasmid) when the control pCMV-LUC was cotransfected (Fig. 2B). There was a moderate (1.5-fold compared to pCMV-LUC) LRH-1-dependent induction of reporter activity ( $\mathrm{pLRE}(\mathrm{E} 3)$ CMV-LUC). When the same 26-bp DNA fragment was dimerized (p2XLRE(E3)-CMV-LUC), stronger induction (about 2-fold) resulted. When tetramerized (p4XLRE(E3)-CMV-LUC), much stronger (about 5-fold) induction was observed, which is suggesting that the putative response elements are indeed functional. To study the effect of one nucleotide mismatch at position $-800(\mathrm{~A})$ in the response elements, we substituted the nucleotide $\mathrm{C}$ for $\mathrm{A}$ to perfectly match the consensus sequence. As expected, stronger induction (2-fold compared to pCMV-LUC) was observed, although it was not very dramatic (pcLRE-CMV- LUC). Therefore, the weak induction observed with the pLRE(E3)-CMV-LUC is considered to be significant, because the induction observed with the consensus LRE was not very strong in our system as well. When only the upstream element of the putative response element was mutated (pm1-LRE(E3)- CMV-LUC), there was no responsiveness to LRH-1 (Fig. 2A \& 2B). A differently mutated LRE (pm2-LRE(E3)-CMV-LUC), which has sequence changes mostly at the downstream element, obliterated responsiveness to the LRH-1 as well. Taken together, we have identified a 16-bp LRH-1 response element in the ECR3 of NPC1L1 gene.

\section{Specific binding of LRH-1 nuclear receptor to the LRH-1 response element in ECR3}

To demonstrate that the LRH-1 response element in ECR3 binds LRH-1, we performed an electrophoretic mobility shift assay (EMSA) with $\mathrm{LRH}-1$ protein produced by in vitro transcription/translation reaction and a ${ }^{32} \mathrm{P}$-labeled 26-bp DNA probe. A strong shifted band was evident when the $\mathrm{LRH}-1$ protein was allowed to bind (Fig. 2C). The shifted band was efficiently competed by a 100-fold molar excess of the unlabeled consensus LRH-1 response element (CLRE) and unlabeled probe $(\operatorname{LRE}(E 3))$. When the $\mathrm{m} 1-\operatorname{LRE}(\mathrm{E} 3)$, which has sequence changes only at the upstream element of the $\mathrm{LRH}-1$ response element, was used as a competitor, the shifted band still competed strongly but not as efficiently as the cLRE and LRE(E3); this is suggesting that the upstream response element is required for maximal binding activity. On the other hand, a 100-fold molar excess of the m2-LRE(E3), which has sequence changes mostly at the downstream element, was not able to compete at all; this is suggesting that the downstream element may play a major role in binding to $\mathrm{LRH}-1$ protein. These results indicate that the $\mathrm{LRH}-1$ protein binds to this response element in a specific manner in vitro. To further examine whether this specific binding was due to $L R H-1$, we included antibodies against $L R H-1$ and $L X R \alpha$ in the binding reaction. Incubation with anti-LRH-1 antibody resulted in a clear supershifted band (Fig. 2C). The control anti-LXR $\alpha$ antibody has no effect on the migration of the shifted band, indicating that the protein bound to the LRE(E3) and CLRE was indeed LRH-1. 
Chromatin immunoprecipitation assay was performed to determine whether LRH-1 is bound to the LRH-1 response element (LREs) of ECR3 in the nuclei of HepG2 cells. As shown in Fig. 2D, LRE-containing ECR3 chromatin fragment from HepG2 cells, which had been transfected with LRH-1 expression plasmid, was enriched by immunoprecipitation with anti-LRH-1 antibody. Two regions of bile salt export pump (BSEP) gene were amplified as positive and negative controls, respectively (12). This result provides evidence that $\mathrm{LRH}-1$ binds to the LRE in ECR3 of the NPC1L1 gene in living HepG2 cells. Taken together, the LRE in ECR3 of the NPC1L1 gene appears to be a functional $L R H-1$ response element.

\section{ECR3 responds to $\mathrm{LRH}-1$ when linked to its natural basal promoter}

We have identified LRH-1 response element in ECR3 by using a heterologous CMV promoter. To study transcriptional regulation of ECR3 by LRH-1 under a more natural condition, we constructed $p(-1380 \sim+51)$-LUC (Wt) which contained both ECR3 and basal promoter of the NPC1L1 gene; then, we made a series of truncations from the upstream end $(-1380)$. When Wt was tested, a dose-dependent induction by LRH-1 was observed (Fig. 3A). As expected, truncation down to -863 $(\mathrm{Wt}-\mathrm{d} 1)$ resulted in a small change in LRH-1-dependent induction, while further truncation down to -775 (Wt-d2) obliterated LRH-1-dependent induction. This demonstrated that the $\mathrm{LRH}-1$ response element in ECR3 is functional when linked to its natural basal promoter. There appears to be no other $\mathrm{LRH}-1$ response elements in the region from -1380 to +51 .

\section{Synergistic transcriptional activation of NPC1L1 gene by \\ LRH-1 and SREBP2}

Previous studies have demonstrated that several transcription factors are responsible for the regulation of NPC1L1 gene. For example, hepatocyte nuclear factor $1 \alpha$ (HNF1 $\alpha$ ), hepatocyte nuclear factor $4 \alpha$ (HNF4 $\alpha$ ), peroxisome proliferator-activated receptor $\alpha$ (PPAR $\alpha)$, and SREBP2 have been shown to regulate NPC1L1 gene (14-17). The LRH-1, which is bound to the response element identified in this study, is very likely to interact with other transcription factors which are already known to be bound to the promoter region. Indeed, moderate additive transcriptional activation resulted when SREBP2, in addition to LRH-1 expression plasmids, was co-transfected with $\mathrm{p}(-1380$ $\sim+51$ )-LUC (Wt, Fig. 3A). However, when other transcription factors, such as HNF4 $\alpha$ and PPAR $\alpha$, were tested in combination with LRH-1 or SREBP2, no further activation was observed (data not shown). To study whether the binding of $\mathrm{LRH}-1$ to LRE is required for the additive activation, we changed nucleotide sequence at the $\mathrm{LRH}-1$ response element by site-directed mutagenesis. With MutA, which contains mutated upstream but intact downstream LRH-1 response elements (Fig. 2A), only weak induction by LRH-1 was observed. With MutB, which contains both upstream and downstream LRH-1 response elements that are mutated, no significant further induction by
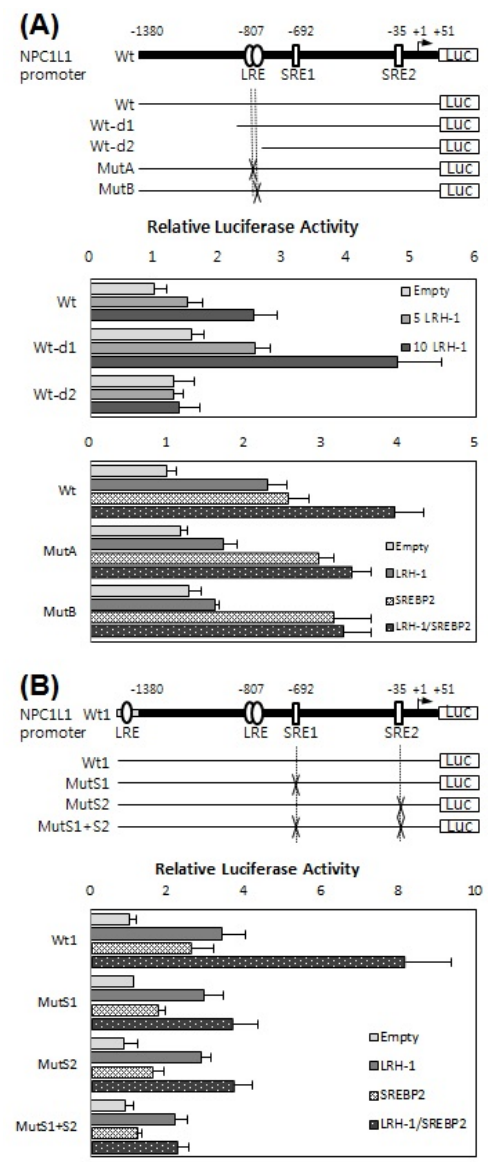

Fig. 3. Synergistic transcriptional activation of NPC1L1 gene by nuclear receptors LRH-1 and SREBP2 is shown. (A) Transcriptional induction of ECR3, which is linked to its natural promoter, by LRH-1 is dependent on the LRE. Mutations were generated by either truncating from the upstream end or changing nucleotides at each LRE, as shown in Fig. 2A, in the context of the $p(-1380 \sim$ +51)-LUC reporter construct (top). Transfection with 5 to $10 \mathrm{ng}$ of LRH-1 expression plasmid was performed, as described in Materials and Methods. Relative luciferase activities are shown (middle), which were calculated by dividing the normalized luciferase activities by that of control cells transfected with Wt and an empty expression plasmid (pcDNA3). Combinations of the following expression plasmids were used for co-transfection. Up to $10 \mathrm{ng}$ of an empty vector, $10 \mathrm{ng}$ of $\mathrm{LRH}-1$ plasmid, and $0.1 \mathrm{ng}$ of SREBP2 plasmid are shown (bottom). Values are expressed as the mean \pm S.E. $(2<n<7)$. (B) Requirement of two functional SREs is shown for synergistic induction of NPC1L1 gene by nuclear receptors LRH-1 and SREBP2. Mutations were generated in each SRE located at the position -692 or -35 , or in both (top). The pint10 $(-1380 \sim+51)$-LUC (Wt1) contains a 276-bp fragment of intron 10 at the upstream end of $p(-1380 \sim+51)$-LUC (Wt).

LRH-1 was observed, when coexpressed with SREBP2. Therefore, binding of $L R H-1$ to the response element is required for additive activation with SREBP2.

While we were searching for sequences matching the con- 
sensus LRH-1 response element in the NPC1L1 gene, we discovered clustered putative LRH-1 response elements in intron 10 , which were not included in the ECRs. When a 276-bp fragment $(+15012 \sim+15287)$ containing three putative LRH-1 response elements were tested using CMV promoter, about 5-fold induction by LRH-1 was observed (data not shown). We inserted this 276-bp fragment at the upstream end of $p$ $(-1380 \sim+51)$-LUC, resulting in Wt1, to test whether there is any improvement in transcriptional activation. As expected, there was a stronger LRH-1-dependent induction (by 3.4-fold) of reporter activity, while there was a small difference in SREBP2-dependent induction (by 2.6-fold). However, synergistic transcriptional induction (by about 8-fold) resulted when both LRH-1 and SREBP2 were expressed, suggesting that multiple $\mathrm{LRH}-1$ response elements in NPC1L1 gene may play important roles in regulating NPC1L1 gene (Fig. 3B). To examine the influence of SREs on the synergistic transcriptional activation of the NC1L1 promoter by LRH-1 and SREBP2, we constructed mutant reporter plasmids in which either or both of the SREs were mutated in the Wt1 (Fig. 3B). With MutS1 and MutS2, which contain one mutated SRE at upstream and downstream SREs, respectively, only the weak induction by SREBP2 was observed. Furthermore, synergistic activation was abolished, suggesting that both of the SREs are required for synergistic activation. When both of the SREs were mutated (MutS1+S2), neither the induction by SREBP2 nor the synergistic activation was observed.

In summary, we have identified LRH-1 response elements in the NPC1L1 gene for the first time, by analyzing evolutionarily conserved regions which are distributed all over $29 \mathrm{~kb}$ region of the NPC1L1 gene. Furthermore, synergistic transcriptional activation suggests that a combination of LRH-1 and SREBP2 may play important roles in regulating the expression of NPC1L1 gene.

\section{MATERIALS AND METHODS}

\section{Recombinant Plasmids}

Cytomegalovirus (CMV) promoter-driven expression plasmids carrying transcription factor genes were constructed by inserting appropriate gene fragments into pcDNA3 (Invitrogen). The expression plasmid pcDNA3-LRH-1 was constructed by inserting a $1.65-\mathrm{kb}$ fragment by PCR amplification of HepG2 cDNA. Primer sequences for the PCR amplification of full-length CDNA are ACTActcgaGTCTTCTAATTCAGATACTG and GCTCCTAGGGGTTGTAACT. The luciferase reporter plasmids, pE-CMV-LUC, were constructed by inserting the appropriate ECR fragments, which were prepared by the PCR amplification of a BAC clone (RP11-1031N24), into the pT-CMV-LUC plasmid (19). Primer pairs for the PCR amplification of ECRs are available upon request. Deletion series were constructed using pE3-CMV-LUC which contains the ECR3 in reverse orientation. pE3-d51-CMV-LUC was constructed by self-ligating a $5.5 \mathrm{~kb}$ fragment after the digestion of pE3-CMV-LUC with Pst I.
pE3-d52-CMV-LUC and pE3-d53-CMV-LUC were constructed by inserting a $0.23 \mathrm{~kb}$ and $0.17 \mathrm{~kb}$ PCR-amplified/Not I-digested fragments into pT-CMV-LUC, respectively. Primer sequences for PCR amplification are available upon request. The CMV-LUCs containing LRH-1 response elements were constructed by inserting annealed oligonucleotides into pTCMV-LUC. Primer sequences are GAGAACCCAAGGTCGGCCTTGa/CAAGGCCGACCTTGGGTTCTCa (pcLRE), AGAACCCAAGGTAGGCCTTGAAGATTa/AATCTTCAAGGCCTAC CTTGGGTTCTa (pLRE(E3)), AGAACCAACGTTAGGCCTTGAAGATTa/AATCTTCAAGGCCTAACGTTGGTTCTa (pm1-LRE(E3)), and AGAACCCAAGGTATGCATGTAAGATTa/ AATCTTACATGCATACCTTGGGTTCTa (pm2-LRE(E3)). The promoter region of the NPC1L1 gene $(-2108 \sim+51)$ was prepared by PCR amplification using AGAAGGAGGAAACATTGTTAGGTG/ GTCTGGGAAGGGGTCAGC. The $p(-1380 \sim$ +51)-LUC (Wt) was prepared by ligating a pair of annealed primers (GGCCGCACTGCAGATGCA/TCTGCAGTGC) with 6.4-kb Not I- Nsi I fragment from $\mathrm{p}(-2108 \sim+51)$-LUC plasmid. The $p(-863 \sim+51)$-LUC (Wt-d1) and $p(-775 \sim$ $+51)$ - LUC (Wt-d2) plasmids were prepared by using Apa I site at -863 and a primer GGAGCAGAAAGTGTGTACCA, respectively. The MutA and MutB were prepared by using GTGATAGTGAGAACCAACGTTAGGCCTTGAAGATT/AATCT TCAAGGCCTAACGTTGGTTCTCACTATCAC and GAGAACCCAAGGTATGCATGTAAGATTCCTATCAGG/CCTGATAGGAATCTTACATGCATACCTTGGGTTCTC, respectively. The pint10 $(-1380 \sim+51)$-LUC(Wt1) was constructed by inserting a 0.28-kb PCR-amplified fragment $(+15012 \sim+15287)$ into $p(-1380 \sim+51)$-LUC. The MutS1 was generated by deleting $4 \mathrm{nt}$ at the $3^{\prime}$-end of the SRE1, using BstE II and mung bean nuclease (CTAGGGGTGAccggt $\rightarrow$ CTAGGGcggt). The MutS2 was generated by site-directed mutagenesis (GAAGGGGAGG $\rightarrow$ GAActcGAGG). Detailed strategies used for constructing the expression and reporter plasmids are available on request.

\section{Cell Culture and Transient Cotransfection Experiments}

HepG2 cells were maintained in Dulbecco's modified Eagle's medium supplemented with $10 \%$ heat-inactivated fetal bovine serum, 100 units $/ \mathrm{ml}$ of penicillin, and $100 \mu \mathrm{g} / \mathrm{ml}$ of streptomycin. The cells were grown at $37^{\circ} \mathrm{C}$ under $5 \% \mathrm{CO}_{2}$. Twentyfour hours before transfection, HepG2 cells were plated at a density of $1.2 \times 10^{5}$ cells per well in a 24 -well plate. As described in the figure legends, each transfection contained 0.1 to $10 \mathrm{ng}$ of the transcription factor expression plasmids, $20 \mathrm{ng}$ of a CMV-luciferase reporter plasmid (200 ng of reporter plasmids without CMV promoter), 50 ng of a control plasmid pCMV-lacZ, and 400 ng (or 200 ng) of pGem1 carrier using $1.2 \mu \mathrm{l}$ of $0.45 \% \mathrm{PEI}$ (Aldrich). All experiments were performed in duplicates. Forty-eight hours after the treatment, the ratio of luciferase/ $\beta$-galactosidase activities were determined.

\section{Quantitative real-time PCR (QRT-PCR)}

Real-time PCRs were performed on the LightCycler Nano re- 
al-time PCR system (Roche). Primer sequences are available upon request. Total RNA from HepG2 cells was extracted with Accuzol (Bioneer, Korea).

\section{Immunoblot}

Protein samples were run on $8 \%$ SDS-PAGE gel and were electrophoretically transferred to a nitrocellulose membrane. Membranes were blocked with $5 \%$ nonfat dry milk in PBS containing $0.1 \%$ Tween for $1 \mathrm{~h}$, and they were incubated for 1 $\mathrm{hr}$ with the antibodies indicated in the figure legends and detected by chemiluminescence.

\section{Electrophoretic mobility-shift assay (EMSA)}

The LRH-1 protein was synthesized using TNT Quick Coupled Transcription/Translation Systems (Promega). The strand sequences for $\mathrm{LRH}-1$ response element (ECR3), the consensus $\mathrm{LRH}-1$ response element, and the mutated fragments $\mathrm{m} 1-$ $\operatorname{LRE}(\mathrm{E} 3)$ and $\mathrm{m} 2-\mathrm{LRE}(\mathrm{E} 3)$ are basically the same as those described in Recombinant Plasmids. Double-stranded oligonucleotides were end-labeled with $\left[\gamma_{-}^{32} \mathrm{P}\right]$ ATP using T4 polynucleotide kinase (NEB). EMSA was performed as described previously (19).

\section{Chromatin immunoprecipitation (ChIP) assay}

HepG2 cells were transfected with 100 ng of $\mathrm{LRH}-1$ expression plasmid and $3.5 \mu \mathrm{g}$ of pGem1 plasmid using a $60-\mathrm{mm}$ dish. ChIP assay was performed as previously described (19), using $20 \mu \mathrm{g}$ of an antibody against LRH-1 (Santa Cruz Biotechnology) by incubating overnight at $4^{\circ} \mathrm{C}$. The immunoprecipitates were analyzed by PCR using the following primers: For ECR3, TTCCTGCAGCTGTCTCCAG/AATCTTCAAGGCCTACCTTGGGTTCT was used; for positive $(-253 \sim+35$ of BSEP gene) and negative ( $-990 \sim-813$ of BSEP gene) controls, GATAGCCTGAATTCCAGGGCTC/TTCACAACCTITTCCAACCTCGG and GCCCATCAATTGCATTTCAGAGC/TCTCAACAGCCCTAGGAGTTGG were used, respectively (12).

\section{ACKNOWLEDGEMENTS}

This research was supported by Hallym University Sepcialization Fund (HRF-S-11) and Priority Research Centers Program grant from the National Research Foundation (NRF-20090093812) funded by the Ministry of Science, ICT \& Future Planning in the Republic of Korea.

\section{REFERENCES}

1. Altmann SW, Davis HR Jr, Zhu LJ et al (2004) Niemann -Pick C1 Like 1 protein is critical for intestinal cholesterol absorption. Science 303, 1201-1204

2. Temel RE, Tang W, Ma Y et al (2007) Hepatic NiemannPick C1-like 1 regulates biliary cholesterol concentration and is a target of ezetimibe. J Clin Invest 117, 1968-1978

3. The Myocardial Infarction Genetics Consortium Investigators (2014) Inactivating Mutations in NPC1L1 and Protec- tion from Coronary Heart Disease. N Engl J Med 371, 2072-2082

4. Garcia-Calvo M, Lisnock J, Bull HG et al (2005) The target of ezetimibe is Niemann-Pick C1-Like 1 (NPC1L1). Proc Natl Acad Sci U S A 102, 8132-8137

5. Stein S, Oosterveer MH, Mataki C et al (2014) SUMOylationDependent LRH-1/PROX1 Interaction Promotes Atherosclerosis by Decreasing Hepatic Reverse Cholesterol Transport. Cell Metab 20, 603-613

6. Fayard E, Auwerx J and Schoonjans K (2004) LRH-1: an orphan nuclear receptor involved in development, metabolism and steroidogenesis. Trends Cell Biol 14, 250-260

7. Schoonjans K, Annicotte JS, Huby T et al (2002) Liver receptor homolog 1 controls the expression of the scavenger receptor class B type I. EMBO Rep 3, 1181-1187

8. Freeman LA, Kennedy A, Wu J et al (2004) The orphan nuclear receptor LRH-1 activates the ABCG5/ABCG8 intergenic promoter. J Lipid Res 45, 1197-1206

9. Delerive P, Galardi CM, Bisi JE, Nicodeme E and Goodwin B (2004) Identification of liver receptor homolog-1 as a novel regulator of apolipoprotein Al gene transcription. Mol Endocrinol 18, 2378-2387

10. Luo Y, Liang CP and Tall AR (2001) The orphan nuclear receptor LRH-1 potentiates the sterol-mediated induction of the human CETP gene by liver X receptor. J Biol Chem 276, 24767-24773

11. Out C, Hageman J, Bloks VW et al (2011) Liver receptor homolog-1 is critical for adequate up-regulation of Cyp7a1 gene transcription and bile salt synthesis during bile salt sequestration. Hepatology 53, 2075-2085

12. Song X, Kaimal R, Yan B and Deng R. (2008) Liver receptor homolog 1 transcriptionally regulates human bile salt export pump expression. J Lipid Res 49, 973-984

13. Matsukuma KE, Wang L, Bennett MK and Osborne TF. (2007) A key role for orphan nuclear receptor liver receptor homologue-1 in activation of fatty acid synthase promoter by liver $X$ receptor. J Biol Chem 282, 20164-71

14. Pramfalk C, Jiang ZY, Cai, Q et al (2010) HNF1alpha and SREBP2 are important regulators of NPC1L1 in human liver. J Lipid Res 51, 1354-1362

15. Iwayanagi Y, Takada T and Suzuki H (2008) HNF4alpha is a crucial modulator of the cholesterol-dependent regulation of NPC1L1. Pharm Res 25, 1134-41

16. Iwayanagi Y, Takada T, Tomura F et al (2011) Human NPC1L1 expression is positively regulated by PPAR $\alpha$. Pharm Res 28, 405-412

17. Alrefai WA, Annaba F, Sarwar Z et al (2007) Modulation of human Niemann-Pick C1-like 1 gene expression by sterol: Role of sterol regulatory element binding protein 2 . Am J Physiol Gastrointest Liver Physiol 292, G369-376

18. Davies JP, Levy B and Ioannou YA (2000) Evidence for a Niemann-Pick C (NPC) gene family: identification and characterization of NPC1L1, Genomics 65, 137-145

19. Back SS, Kim J, Choi D, Lee ES, Choi SY and Han K (2013) Cooperative transcriptional activation of ATP-binding cassette sterol transporters ABCG5 and ABCG8 genes by nuclear receptors including Liver-X-Receptor. BMB Rep 46, 322-327

20. Fayard E, Schoonjans K, Annicotte JS and Auwerx J (2003) Liver receptor homolog 1 controls the expression of carboxyl ester lipase. J Biol Chem 278, 35725-35731 